\title{
BMJ Open Rates and drivers of progression to pre-diabetes and diabetes mellitus among HIV-infected adults on antiretroviral therapy: a global systematic review and meta-analysis protocol
}

\author{
Jobert Richie N Nansseu, ${ }^{1,2}$ Jean Joel R Bigna, ${ }^{3}$ Arnaud D Kaze, ${ }^{4}$ \\ Jean Jacques N Noubiap ${ }^{5,6}$
}

To cite: Nansseu JRN, Bigna JJR, Kaze AD, et al. Rates and drivers of progression to pre-diabetes and diabetes mellitus among HIV-infected adults on antiretroviral therapy: a global systematic review and metaanalysis protocol. BMJ Open 2016:6:e012852.

doi:10.1136/bmjopen-2016012852

- Prepublication history and additional material is available. To view please visit the journal (http://dx.doi.org/ 10.1136/bmjopen-2016012852).

Received 27 May 2016 Revised 25 July 2016 Accepted 18 August 2016

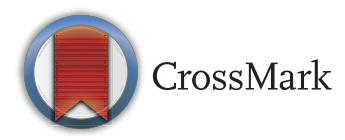

For numbered affiliations see end of article.

Correspondence to Dr Jobert Richie N Nansseu; jobertrichie_nansseu@yahoo.fr

\section{ABSTRACT}

Introduction: With the new 'test and treat' policy of the WHO, it is obvious that the number of HIV-infected patients taking antiretroviral therapy (ART) will grow exponentially, with consequential increase in the burden of diabetes mellitus (DM). Our aim is to summarise existing data on the incidence of prediabetes and DM, and associated risk factors among HIV-infected adults.

Methods and analysis: This systematic review will include cohort studies reporting the incidence of prediabetes and/or DM, and associated risk factors among HIV-infected adults on ART, with these patients being free of any impaired glucose metabolism at study baseline. We will perform electronic searches in PubMed, Excerpta Medica Database (EMBASE), Web of Science and WHO Global Health Library, supplemented with manual searches. Articles published from 1 January 2000 to 31 July 2016, in English or French languages, and without any geographical restriction will be eligible for inclusion. 3 authors will independently screen, select studies, extract data and assess the risk of bias with discrepancies resolved by consensus. We will assess clinical heterogeneity by examining the study design and setting, criteria and cut-offs used to define prediabetes or DM, process of calculation of incidence and outcomes in each study. We will also assess statistical heterogeneity using the $\chi^{2}$ test of homogeneity and quantify it using the $\mathrm{I}^{2}$ statistic. A random effects meta-analysis will be used to estimate the overall cumulative incidence of pre-diabetes/DM and risk factors.

Ethics and dissemination: This systematic review will use data from published studies and does not require ethics approval. Its results are expected to help putting in place action plans and preventive measures to curb the growing burden of DM in the HIV population on ART. Findings will be published in a peer-reviewed journal and presented at scientific conferences.

\section{Strengths and limitations of this study}

To the best of our knowledge, this is the firs systematic review and meta-analysis to determine the incidence of pre-diabetes and diabetes, and the associated risk factors among HIV-infected adults on antiretroviral therapy.

- We will perform an exhaustive literature search to include all potential studies fulfilling the inclusion criteria.

- Likewise, we will use robust analytical methods to carefully examine the research questions with tools dedicated to the meta-analysis of cohort studies. We will also carefully assess risk of bias, and clinical and statistical heterogeneities.

- It is anticipated that all studies may not have calculated the incidence per person-time of follow-up, rending difficult a clear estimate of rates of progression. Additionally, the tests used to investigate the risk factors may differ from one study to another, affecting pooling of findings from each study.

PROSPERO registration number: CRD42016039651.

\section{INTRODUCTION}

The advent and widespread use of highly active antiretroviral therapy (HAART) has been a success story in the history and evolution of the HIV pandemic. Indeed, it has significantly improved the longevity, prognosis and quality of life of people suffering from the disease. However, the flipside is that as life expectancy increases and the duration of exposure to antiretroviral therapy (ART) 
lengthens, hence these patients are more likely to develop numerous complications mainly because of mitochondrial toxicity, such as hyperlactatemia, lactic acidosis, pancreatitis, lipodystrophy and dysglycaemia. ${ }^{1-5}$

Pre-diabetes, which is characterised by impaired fasting glucose (IFG) and/or impaired glucose tolerance (IGT), ${ }^{7}$ and diabetes mellitus (DM) are growing in importance among the HIV population. There is evidence showing that the prevalence and risk of DM is higher in the HIV population than in the general population; ${ }^{8}$ the risk increasing with use of ART. ${ }^{9}{ }^{10}$ By mid-2015, globally 15.8 million people with HIV were receiving ART; ${ }^{11}$ with the new 'test and treat' policy of the $\mathrm{WHO},{ }^{12}$ it is expected that this number will grow exponentially in the near future, hence a concurrent increase in the number of those progressing to prediabetes and DM, which already cause serious concerns across the globe. ${ }^{13}$

There is therefore an urgent need to think at what can be done to prevent or adequately manage such an upcoming public health catastrophe. How many HIV-infected persons taking ART are susceptible to shift from normal glucose tolerance through pre-diabetes to overt $\mathrm{DM}$ and at what rates? What are the factors driving these conversions? These are some questions needing urgent attention if we want to prevent, delay or adequately respond to the rapidly and continuously increasing burden of DM in the HIV-infected population and in the general population as well.

To date and to the best of our knowledge, there is no study that has summarised the globally existing data on the rates and drivers of progression from normoglycaemia to pre-diabetes and/or DM, and the associated risk factors among the HIV-infected population on ART. The current protocol is for a systematic review and meta-analysis of studies published from 1 January 2000 to 31 July 2016 in order to determine the incidence of pre-diabetes and DM and associated risk factors among HIV-infected adult patients on ART.

\section{METHODS AND ANALYSIS}

We will use the Preferred Reporting Items for Systematic Reviews and Meta-Analyses (PRISMA) statement released in 2009 as guidance to report this review. ${ }^{14}$ This protocol is presented with regard to the PRISMA-P 2015 guidelines, ${ }^{15}$ and registered with PROSPERO (ID=CRD42016039651). The PRISMA-P checklist is available as online supplemental table S1.

\section{Eligibility criteria}

We will systematically identify and appraise reports of original peer-reviewed publications of cohort studies (either retrospective or prospective) which have assessed the incidence of pre-diabetes (including IFG and/or IGT) and/or overt DM, and associated risk factors whenever available. The population of interest will be HIV-infected adult patients aged 18 years and above, initiating or already on ART, with proven normal glucose tolerance before inclusion in the cohort. The criteria used to define pre-diabetes or DM must have been clearly worded in the studies. Studies must have been released from 1 January 2000 to 31 July 2016 and reported either in English or French languages. No geographical restriction will be applied. Studies conducted among HIV-infected pregnant women will not be considered in this review. Likewise, case-control and crosssectional studies, letters, reviews, commentaries, editorials, case reports or case series will not be included as well. For duplicate reports, the most comprehensive and up-to-date version will be taken into account.

\section{Information sources}

Extensive electronic searches will be performed in PubMed/MEDLINE, Excerpta Medica Database (EMBASE), ISI Web of Science (Science Citation Index), and the WHO Global Health Library. We will supplement this literature search by scanning the reference lists of relevant studies and other relevant review articles, specialist journals and conference proceedings. Besides major well-known HIV cohorts will be contacted for additional information (either published or not), if existent.

\section{Search strategy and selection of studies}

A comprehensive search of the peer-reviewed and grey literature will be conducted to identify all appropriate studies available from 1 January 2000 to 31 July 2016 and fulfilling our inclusion criteria. The strategy used to run the PubMed search is shown in table 1 . For other database searches, we will develop a combination using terms like: "human immunodeficiency virus", "acquired immunodeficiency syndrome", "antiretroviral therapy", "incidence", "cohort", "prediabetes", "hyperglycemia", "dysglycemia" or "diabetes".

Willing to make sure that inclusion criteria will be adhered to and consistently applied by all review authors, we will develop and pilot a screening guide. Three review authors (JRNN, JJRB and ADK) will independently review all retrieved citations on the basis of titles and abstracts. Thereafter, the same authors will independently assess the full-texts of records deemed relevant or potentially relevant for eligibility. Agreement between review authors will be measured using the Cohen's $\kappa$-statistic. ${ }^{16}$ Disagreements will be resolved through discussion and consensus, or arbitration by another author (JJNN).

\section{Data abstraction}

A preconceived and standardised abstraction form will be used to extract and collect the data, conducted by three independent review authors (JRNN, ADK and JJRB). Disagreements between these authors will be reconciled through discussion and consensus. Data will be retrieved from each study for the following elements: title, first author, year of publication, period of study, 


\section{Table 1 PubMed search strategy}

\begin{tabular}{lll} 
Number & Query & Result \\
\hline$\# 1$ & (HIV) OR (AIDS) & 403080 \\
$\# 2$ & $\begin{array}{l}\text { (antiretroviral therapy) OR (highly active antiretroviral therapy) OR (antiretroviral treatment) OR } \\
\text { (highly active antiretroviral treatment) OR (ART) OR (HAART) OR (cART) }\end{array}$ \\
& $\begin{array}{l}\text { (diabetes) OR (prediabetes) OR (hyperglycemia) OR (glucose intolerance) OR (disturbed glucose } \\
\text { metabolism) OR (dysglycemia) OR (impaired fasting glucose) OR (impaired glucose tolerance) OR } \\
\end{array}$ & (impaired glucose metabolism) \\
$\# 4$ & (incidence) OR (incident) OR (cohort) \\
$\# 5$ & \#1 AND \#2 AND \#3 AND \#4 \\
L6 & Limits: from 2000/01/01 to 2016/07/31
\end{tabular}

country(ies) and region(s) of origin, objective(s) and study design, study end point(s), study population, criteria used to define pre-diabetes or DM, size of the study population, age of participants (range and mean or median), male/female ratio, duration of follow-up (mean or median), ART regimen, mean duration of ART, incidence of pre-diabetes and/or DM, risk factors (after adjustments) and study conclusion(s). For missing data, authors of included studies will be contacted.

\section{Risk of bias in individual studies}

The Newcastle-Ottawa Scale (NOS) for cohort studies will serve to evaluate the methodological quality of studies included in this review. ${ }^{17}$ This scale is primarily formulated by a star allocation system, assigning a maximum of nine stars for the risk of bias in three areas: selection of study groups (4 stars), comparability of groups (2 stars), and ascertainment of the outcome of interest (3 stars). There is no validation study that provides a cut-off score for rating low-quality studies; a priori, we arbitrarily established that $0-3,4-6$ and 7-9 stars will be considered high, moderate and low risk of bias, respectively.

We will provide a thorough description of the missing data and dropouts for each included study, and the extent to which these missing data could have influenced the study results. Additionally, we will examine whether all outcomes have been reported and reasons behind any exclusion. Three researchers will independently assess the quality of studies included, and resolve any differences by discussion and consensus. We will use the Cohen's $\kappa$-coefficient to calculate the degree of agreement between investigators and measure the interrater agreement. ${ }^{16}$

\section{Data synthesis including assessment of heterogeneity}

Data will be analysed using STATA V. 14 (Stata Corp, Texas, USA). We will present a table of the main characteristics of included studies and a summary flow diagram for potentially eligible studies that were subsequently excluded, and reasons for exclusion. Range, mean (SD) or median (range), and frequency (percentage) will serve to summarise data when appropriate.
Clinical heterogeneity will be investigated by examining the study design and setting (including geographical region), criteria and cut-offs used to define pre-diabetes or DM, the process of calculation of incidence, and outcomes in each study. The main outcomes of interest are the incidence of dysglycaemias (pre-diabetes and/or $\mathrm{DM}$ ) in the first place, and potential factors of conversion (from normal glucose tolerance to either prediabetes or DM) in the second place. Forest plots will be drawn to visualise the combined cumulative incidence, risk factors and the extent of statistical heterogeneity between studies. The $\chi^{2}$ test on Cochrane's $Q$ statistic will serve to assess statistical heterogeneity, ${ }^{18}$ quantified by calculating the $\mathrm{I}^{2}$ statistic (with values of $25 \%, 50 \%$ and $75 \%$ being representative of low, medium and high heterogeneity, respectively). ${ }^{19}$ If the $\mathrm{I}^{2}$ statistic is $<50 \%$ and the $p$ value for the test of heterogeneity $\geq 0.1$, studies will be considered homogenous and a fixed-effects meta-analysis will be used to estimate the overall cumulative incidence of pre-diabetes or DM, and the potential driving factors; if not, a random-effects meta-analysis will be undertaken. ${ }^{20}$ Likewise still in case of statistical heterogeneity, subgroup analyses will be performed to explore the potential reasons for heterogeneity. These subgroup analyses will be conducted based on clinical parameters, geographical region and methodological quality of the study. Meta-regression analyses will be undertaken to explain the predictors of the effect size. Nonetheless, if the included studies are too heterogeneous to be pooled together, we will summarise their findings in a narrative format.

Publication bias will be assessed with funnel plots, complemented with the use of Egger's test of bias. Additionally, we will apply the trim-and-fill method to assess the impact of potential publication bias. ${ }^{16}{ }^{21} \mathrm{~A} \mathrm{p}$ value $<0.05$ will be considered indicative of statistically significant publication bias.

\section{Ethics and dissemination}

This systematic review does not require ethical approval since it is based on published studies and not individual participant's data. Findings from this review are expected to have significant clinical and public health impacts. First and foremost, it will inform on the rates of 
progression to DM in HIV-infected adult patients on ART, with consequential anticipated information on the potential number of HIV-infected patients who might get into overt DM and plan the resources that have to be put in place for their management. On the other hand, knowing the factors driving this progression towards DM will permit to develop and implement a package of intervention measures willing to delay or stop this progression, and reduce the burden of the disease and associated complications. Moreover, we will conduct comparisons with the general population to see whether the projected burden of pre-diabetes and DM would be more severe among the HIV population on ART or not. The results of this systematic review and meta-analysis will be published in a peer-reviewed journal and shared at relevant scientific conferences.

\section{Author affiliations}

${ }^{1}$ Department of Public Health, Faculty of Medicine and Biomedical Sciences, University of Yaoundé I, Yaoundé, Cameroon

${ }^{2}$ Sickle Cell Disease Unit, Mother and Child Center, Chantal Biya Foundation, Yaoundé, Cameroon

${ }^{3}$ Department of Epidemiology and Public Health, Centre Pasteur of Cameroon, Yaoundé, Cameroon

${ }^{4}$ Harvard T.H Chan School of Public Health, Boston, Massachusetts, USA ${ }^{5}$ Department of Medicine, Groote Schuur Hospital and University of Cape Town, Cape Town, South Africa

${ }^{6}$ Medical Diagnostic Center, Yaoundé, Cameroon

Contributors JRNN conceived, designed and drafted the protocol. JJRB, ADK and JJNN critically reviewed and revised the manuscript for methodological and clinical content. All the authors approved the final manuscript.

Competing interests None declared.

Provenance and peer review Not commissioned; externally peer reviewed.

Open Access This is an Open Access article distributed in accordance with the Creative Commons Attribution Non Commercial (CC BY-NC 4.0) license, which permits others to distribute, remix, adapt, build upon this work noncommercially, and license their derivative works on different terms, provided the original work is properly cited and the use is non-commercial. See: http:// creativecommons.org/licenses/by-nc/4.0/

\section{REFERENCES}

1. Kiertiburanakul S, Luengroongroj $P$, Sungkanuparph $S$. Clinical characteristics of HIV-infected patients who survive after the diagnosis of HIV infection for more than 10 years in a resourcelimited setting. J Int Assoc Physicians AIDS Care (Chic) 2012;11:361-5

2. Mocroft A, Reiss P, Gasiorowski J, et al. Serious fatal and nonfatal non-AIDS-defining illnesses in Europe. J Acquir Immune Defic Syndr 2010;55:262-70.

3. Hasse $\mathrm{B}$, Ledergerber $\mathrm{B}$, Furrer $\mathrm{H}$, et al. Morbidity and aging in HIV-infected persons: the Swiss HIV cohort study. Clin Infect Dis 2011;53:1130-9.

4. Pefura Yone EW, Kengne AP, Ashuntantang G, et al. Dyslipidaemia in HIV-1-infected patients receiving protease inhibitors after initial treatment with first-line-based non-nucleoside reverse transcriptase inhibitors: a cross-sectional study. BMJ Open 2012;2:pii:e001317.

5. Mamiafo CT, Moor VJ, Nansseu JR, et al. Hyperlactatemia in a group of HIV patients living in Yaounde-Cameroon. AIDS Res Ther 2014;11:2.

6. American Diabetes Association. Diagnosis and classification of diabetes mellitus. Diabetes Care 2013;36(Suppl 1):S67-74.

7. Buysschaert M, Bergman M. Definition of prediabetes. Med Clin North Am 2011:95:289-97. vii.

8. Galli L, Salpietro S, Pellicciotta G, et al. Risk of type 2 diabetes among HIV-infected and healthy subjects in Italy. Eur J Epidemiol 2012;27:657-65.

9. Isa SE, Oche AO, Kang'ombe AR, et al. Human immunodeficiency virus and risk of type 2 diabetes in a large adult cohort in Jos, Nigeria. Clin Infect Dis 2016;63:830-5.

10. Levitt NS, Peer N, Steyn K, et al. Increased risk of dysglycaemia in South Africans with HIV; especially those on protease inhibitors. Diabetes Res Clin Pract 2016;119:41-7.

11. World Health Organization. HIV/AIDS Fact sheet $N^{\circ} 360$. Geneva: WHO. 2015.

12. World Health Organization. Guideline on when to start antiretrovira therapy and on pre-exposure prophylaxis for HIV. Geneva: World Health Organization, 2015:78.

13. International Diabetes Federation. IDF Diabetes Atlas. 7th edn Brussels, Belgium: International Diabetes Federation, 2016

14. Moher D, Liberati A, Tetzlaff J, et al. PRISMA Group. Preferred Reporting Items for Systematic Reviews and Meta-Analyses: the PRISMA statement. J Clin Epidemiol 2009;62:1006-12.

15. Moher D, Shamseer L, Clarke M, et al. Preferred Reporting Items for Systematic Review and Meta-Analysis Protocols (PRISMA-P) 2015 statement. Syst Rev 2015;4:1.

16. Viera AJ, Garrett JM. Understanding interobserver agreement: the kappa statistic. Fam Med 2005;37:360-3.

17. Wells G, Shea B, Peterson J, et al. The Newcastle-Ottawa Scale (NOS) for assessing the quality of nonrandomised studies in meta-analyses. http://www.ohri.ca/programs/clinical_epidemiology/ oxford.asp (accessed 26 Jan 2016).

18. Cochran GW. The combination of estimates from different experiments. Biometrics 1954;10:101-29.

19. Higgins JP, Thompson SG. Quantifying heterogeneity in a meta-analysis. Stat Med 2002;21:1539-58.

20. Higgins JP, Thompson SG, Deeks JJ, et al. Measuring inconsistency in meta-analyses. BMJ 2003;327:557-60.

21. Duval S, Tweedie R. Trim and fill: a simple funnel-plot-based method of testing and adjusting for publication bias in meta-analysis. Biometrics 2000:56:455-63. 